\title{
From lattice Quantum Electrodynamics to the distribution of the algebraic areas enclosed by random walks on $\mathbb{Z}^{2}$
}

\author{
Thomas Epelbaum ${ }^{1 *}$, François Gelis ${ }^{2 \dagger}$, Bin $\mathrm{Wu}^{2 \ddagger}$
}

October 1, 2018

1. McGill University, Department of Physics 3600 University Street, Montreal QC H3A 2T8, Canada

2. Institut de physique théorique, Université Paris Saclay CEA, CNRS, F-91191 Gif-sur-Yvette, France

\begin{abstract}
In the worldline formalism, scalar Quantum Electrodynamics on a 2dimensional lattice is related to the areas of closed loops on this lattice. We exploit this relationship in order to determine the general structure of the moments of the algebraic areas over the set of loops that have fixed number of edges in the two directions. We show that these moments are the product of a combinatorial factor that counts the number of such loops, by a polynomial in the numbers of steps in each direction. Our approach leads to an algorithm for obtaining explicit formulas for the moments of low order.
\end{abstract}

MSC : 05A15

Key words : random walks on $\mathbb{Z}^{2}$, algebraic areas, lattice QED

\section{Introduction}

In a recent work [1, we have studied the short distance behavior of the one-loop expectation value of local operators in lattice scalar quantum chromodynamics. For this study, we used a discrete version of the worldline formalism [2, 3, 4, 5, in which propagators are represented as a sum over all the random walks on the lattice, that connect the two endpoints of the propagator. In this approach, the

\footnotetext{
*tomepel@gmail.com

†francois.gelis@cea.fr (corresponding author)

¥bin.wu@cea.fr
} 
coefficients of the expansion in powers of the lattice spacing are related to combinatorial properties of these random walks. The leading coefficient merely counts the random walks of a certain length, but the following coefficients are related to areas enclosed by the random walk (for local operators, the two endpoints of the propagator are at the same point, and therefore its worldline representation is a sum over closed random walks). In fact, the successive terms of this expansion can be related to the moments of the distribution of the areas. In the case of an isotropic lattice (i.e. with identical lattice spacings in all directions), the results we needed could be found in ref. [6], in which the authors obtain the general structure of the moments of the area distribution over the set of closed random walks of fixed length $2 n$. Moreover, this paper contains explicit formulas for the moments of order 2 and 4, that were sufficient for our purposes in the isotropic case.

However, the generalization of this expansion to the case of an anisotropic lattice (i.e. with different lattice spacings in each direction) requires the moments of the area over the set of closed random walks that make $n_{1}$ steps in the direction +1 and $n_{2}$ steps in the direction +2 (and also $n_{1}, n_{2}$ steps in the directions $-1,-2$ ). Some numerical explorations and guesswork suggested a very simple form for these moments, namely the product of the number of such closed walks times a polynomial in $n_{1}, n_{2}$. Then, by taking this structure for granted, we obtained the expressions of these polynomials for the moments $2,4,6,8$ and 10 by making an exhaustive list of all the closed random walks up to the length $2 n=20$. The purpose of the present paper is to prove these formulas, by exploiting the relationship between the distribution of areas and Quantum Electrodynamics (QED) on a 2-dimensional lattice.

Let us consider an infinite planar square lattice, and denote by $\boldsymbol{\Gamma}_{n_{1}, n_{2}}$ the set of the closed loops (with a fixed base point to avoid considering loops that are identical up to a translation) drawn on the edges of this lattice, that make $2 n_{1}$ hops in the directions \pm 1 and $2 n_{2}$ hops in the directions \pm 2 . We are interested in the distribution of the algebraic areas enclosed by the loops in $\boldsymbol{\Gamma}_{n_{1}, n_{2}}$. In this paper, we prove that

$$
\sum_{\gamma \in \boldsymbol{\Gamma}_{n_{1}, n_{2}}}(\text { Area }(\gamma))^{2 l}=\frac{\left(2\left(n_{1}+n_{2}\right)\right) !}{n_{1} !^{2} n_{2} !^{2}} \mathcal{P}_{2 l}\left(n_{1}, n_{2}\right),
$$

where $\mathcal{P}_{2 l}$ is a symmetric polynomial in $n_{1}, n_{2}$ of degree $2 l$. Note that the combinatorial factor $\left(2\left(n_{1}+n_{2}\right)\right) ! /\left(n_{1} !^{2} n_{2} !^{2}\right)$ is nothing but the cardinal of the set $\boldsymbol{\Gamma}_{n_{1}, n_{2}}$. Therefore, the polynomial $\mathcal{P}_{2 l}\left(n_{1}, n_{2}\right)$ can be interpreted as the mean value of $(\text { Area }(\gamma))^{2 l}$ over this set. Our approach provides an algorithm for calculating these polynomials explicitly. The first two non-zero moments 
involve the following polynomials,

$$
\begin{aligned}
& \mathcal{P}_{2}\left(n_{1}, n_{2}\right)=\frac{n_{1} n_{2}}{3}, \\
& \mathcal{P}_{4}\left(n_{1}, n_{2}\right)=\frac{n_{1} n_{2}\left(7 n_{1} n_{2}-\left(n_{1}+n_{2}\right)\right)}{15} .
\end{aligned}
$$

(The polynomials that enter in eq. (11) up to $2 l \leq 12$ are listed at the end of the section 3 .)

The rest of this paper is devoted to a proof of eqs. (11) and (2). Our approach is based on the formulation of lattice scalar quantum electrodynamics in the worldline formalism. When this formulation is used in two dimensions, in the presence of a transverse magnetic field, the propagator of the scalar particle can be viewed as a generating function for the distribution of the areas of random loops on a square lattice. In the section 2, we define more precisely the model and recall this correspondence. We also explain how to perform an expansion in powers of the interactions with the magnetic field. In the section 3 , we prove the main result, namely eq. (11). Our proof leads to an algorithm to obtain explicit expressions for the polynomial $\mathcal{P}_{2 l}$, that we have implemented in order to obtain the formulas (2) and several higher moments. A few intermediate calculations are explained in the appendix $\mathrm{A}$, and some basic links between our approach and the Hofstadter-Harper Hamiltonian are outlined in the appendix B]

\section{Lattice scalar QED in two dimensions}

\subsection{Area distribution of random walks from lattice QED}

Consider the following operator, acting on complex valued functions on $\mathbb{Z}^{2}$ :

$$
\begin{gathered}
\sum_{(k, l) \in \mathbb{Z}^{2}} D_{i j, k l} f_{k l} \equiv \frac{2 f_{i j}-U_{1, i j} f_{i+1 j}-U_{1, i-1 j}^{*} f_{i-1 j}}{a_{1}^{2}} \\
+\frac{2 f_{i j}-U_{2, i j} f_{i j+1}-U_{2, i j-1}^{*} f_{i j-1}}{a_{2}^{2}} .
\end{gathered}
$$

In Quantum Electrodynamics, this operator is the square of the discrete 2dimensional covariant derivative $2^{2}$ in two dimensions, acting on a scalar field. Here, we choose an anisotropic lattice, with lattice spacings $a_{1}$ and $a_{2}$ in the two directions. The coefficients $U_{1, i j}$ and $U_{2, i j}$ are complex phases called "link

\footnotetext{
${ }^{1}$ By summing these expressions over all $n_{1}+n_{2}=n$, we recover the results of ref. [6],

$$
\begin{aligned}
& \sum_{n_{1}+n_{2}=n} \frac{\left(2\left(n_{1}+n_{2}\right)\right) !}{n_{1} !^{2} n_{2} !^{2}} \frac{n_{1} n_{2}}{3}=\left(\begin{array}{c}
2 n \\
n
\end{array}\right)^{2} \frac{n^{2}(n-1)}{6(2 n-1)} \\
& \sum_{n_{1}+n_{2}=n} \frac{\left(2\left(n_{1}+n_{2}\right)\right) !}{n_{1} !^{2} n_{2} !^{2}} \frac{n_{1} n_{2}\left(7 n_{1} n_{2}-\left(n_{1}+n_{2}\right)\right)}{15}=\left(\begin{array}{c}
2 n \\
n
\end{array}\right)^{2} \frac{n^{3}(n-1)\left(7 n^{2}-18 n+13\right)}{60(2 n-1)(2 n-3)} .
\end{aligned}
$$
}

${ }^{2}$ When $U_{1, i j}=1$ and $U_{2, i j}=1$, this operator is just the opposite of the discrete Laplacian. 
variables". Their name comes from the fact that they live on the edges of the lattice, while the function $f_{i j}$ lives on the nodes of the lattice. The link variables have a direction and an orientation: in the notation $U_{1, i j}$, the index 1 is the direction of the link, and the coordinates $i j$ denote the starting point of the oriented link. Thus, $U_{1, i j}$ lives on the edge that connects the points $(i, j)$ and $(i+1, j)$. The complex conjugate of a link variable lives on the same link but has the opposite orientation.

An interesting object is the inverse $G_{i j, k l}$ of this operator, which in QED is nothing but the propagator of a particle in a given external electromagnetic field. It is defined by

$$
\sum_{(k, l) \in \mathbb{Z}^{2}} D_{i j, k l} G_{k l ; m n}=\delta_{i m} \delta_{j n}
$$

In the worldline formalism, this inverse can be written as a sum over closed random walks (see ref. 11). When evaluated with identical endpoints (we take the point of coordinates $(0,0)$, but this choice is irrelevant), its expression reads

$$
G_{00,00}=\frac{\mathbf{a}^{2}}{4} \sum_{n_{1}, n_{2}=0}^{\infty}\left(\frac{h_{1}}{4}\right)^{2 n_{1}}\left(\frac{h_{2}}{4}\right)^{2 n_{2}} \sum_{\gamma \in \boldsymbol{\Gamma}_{n_{1}, n_{2}}} \prod_{\ell \in \gamma} U_{\ell},
$$

where $\prod_{\ell \in \gamma} U_{\ell}$ denotes the product of the link variables encountered along the closed path $\gamma$. We have also defined

$$
\frac{2}{\mathbf{a}^{2}} \equiv \frac{1}{a_{1}^{2}}+\frac{1}{a_{2}^{2}} \quad, \quad h_{1,2} \equiv \frac{\mathbf{a}^{2}}{a_{1,2}^{2}} .
$$

By using an anisotropic lattice, we can isolate closed random walks with a given number of steps in each direction by selecting a given term in the Taylor series of the left hand side in powers of $h_{1}$ and $h_{2}$. In other words, the order in $h_{1}$ and $h_{2}$ counts the numbers of steps made by the random walk in the directions \pm 1 and \pm 2 . In order to obtain an explicit connection to the area enclosed by the paths, we need to specialize the link variables in such a way that they represent a magnetic field transverse to the plane of the lattice. By Gauss's law, the product $\prod_{\ell \in \gamma} U_{\ell}$ will then become the exponential of the magnetic flux through the loop $\gamma$, and will give direct access to the area. For a given transverse magnetic field $B$, there are in fact infinitely many ways to represent it in terms of link variables, due to the gauge invariance of electrodynamic 3 . The choice that we adopt in the rest of this paper is Landau gauge :

$$
U_{1, i j} \equiv 1 \quad, \quad U_{2, i j} \equiv e^{i B a_{1} a_{2} i}
$$

It is easy to check that

$$
\prod_{\ell \in \gamma} U_{\ell}=e^{i B a_{1} a_{2} \operatorname{Area}(\gamma)}
$$

\footnotetext{
${ }^{3}$ Different gauge choices correspond to different ways of writing the algebraic area inside a closed loop $\gamma$ as a contour integral. The Landau gauge used in this paper amounts to using the formula $\oint_{\gamma} x d y$. Gauge transformations change the integrand into $x d y+d \theta(x, y)$, which leaves the area unchanged since the added term is a total derivative.
} 
where Area $(\gamma)$ is the algebraic area enclosed by the path $\gamma$, measured as a number of elementary lattice plaquettes. Note that this equation would be true with any other gauge equivalent choice for the link variables 4 , since the left hand side is a gauge invariant quantity. In the rest of the paper, we denote $\phi \equiv B a_{1} a_{2}$ the magnetic flux through one elementary plaquette of the lattice.

Knowing the left hand side of eq. (5) as a function of $\phi, h_{1,2}$ would therefore give access to the full distribution of areas, since we can write

$$
\sum_{\gamma \in \boldsymbol{\Gamma}_{n_{1}, n_{2}}} e^{i \phi \text { Area }(\gamma)}=\sum_{l=0}^{\infty} \frac{(-1)^{l}}{(2 l) !} \phi^{2 l} \sum_{\gamma \in \boldsymbol{\Gamma}_{n_{1}, n_{2}}}(\text { Area }(\gamma))^{2 l} .
$$

Only the even moments are non-zero, since one gets the opposite algebraic area by reversing the path $\gamma$. Note that this correspondence is equivalent to the well-known relationship between the distribution of areas and the traces of the powers of the Hofstadter-Harper Hamiltonian [7, 8, 9.

\subsection{Rules for the expansion in powers of the magnetic field}

In terms of the rescaled lattice spacings defined in eq. (6), the operator $D$ reads

$$
\mathbf{a}^{2} \sum_{(k, l) \in \mathbb{Z}^{2}} D_{i j, k l} f_{k l} \equiv 4 f_{i j}-h_{1}\left(f_{i+1 j}+f_{i-1 j}\right)-h_{2}\left(e^{i \phi i} f_{i j+1}+e^{-i \phi i} f_{i j-1}\right),
$$

where we have now specialized to the gauge choice of eq. (7). This operator can be separated into a vacuum part and a term due to the interactions with the magnetic field,

$$
D=D^{(0)}-\Delta,
$$

with

$$
\begin{aligned}
& \mathbf{a}^{2} \sum_{(k, l) \in \mathbb{Z}^{2}} D_{i j, k l}^{(0)} f_{k l} \equiv 4 f_{i j}-h_{1}\left(f_{i+1 j}+f_{i-1 j}\right)-h_{2}\left(f_{i j+1}+f_{i j-1}\right) \\
& \mathbf{a}^{2} \sum_{(k, l) \in \mathbb{Z}^{2}} \Delta_{i j, k l} f_{k l} \equiv h_{2}\left(\left(e^{i \phi i}-1\right) f_{i j+1}+\left(e^{-i \phi i}-1\right) f_{i j-1}\right) .
\end{aligned}
$$

Let us first consider the vacuum case, in order to establish the notations. We consider an infinite lattice. In this case, the propagator can be conveniently represented in Fourier space by the following formula,

$$
G_{i j, k l}^{(0)}=\mathbf{a}^{2} \int_{0}^{2 \pi} \frac{d p_{1} d p_{2}}{(2 \pi)^{2}} \frac{e^{i\left(p_{1}(i-k)+p_{2}(j-l)\right)}}{4-2\left(h_{1} \cos \left(p_{1}\right)+h_{2} \cos \left(p_{2}\right)\right)} .
$$

By evaluating this expression with equal endpoints, $i=k, j=l$, and expanding in powers of $h_{1,2}$, we can easily recover eq. (5) for a vanishing flux $\phi=0$. For

\footnotetext{
${ }^{4}$ This is trivially seen by recalling that gauge transformations of the link variables read

$$
U_{1, i j} \rightarrow \Omega_{i j} U_{1, i j} \Omega_{i+1 j}^{*} \quad, \quad U_{2, i j} \rightarrow \Omega_{i j} U_{2, i j} \Omega_{i j+1}^{*} .
$$
}


this, we only need to recall the number of random loops that make $n_{1}$ hops in the direction +1 and $n_{2}$ hops in the direction +2 :

$$
\sum_{\gamma \in \boldsymbol{\Gamma}_{n_{1}, n_{2}}} 1=\frac{\left(2\left(n_{1}+n_{2}\right)\right) !}{n_{1} !^{2} n_{2} !^{2}} .
$$

In order to go beyond this trivial result, we must include the effect of the interaction term $\Delta$. The inverse of eq. (11) can be written as a formal series

$$
G=G^{(0)}+\underbrace{G^{(0)} \Delta G^{(0)}}_{G^{(1)}}+\underbrace{G^{(0)} \Delta G^{(0)} \Delta G^{(0)}}_{G^{(2)}}+\cdots=\sum_{k=0}^{\infty} G^{(k)} .
$$

Note that this is not exactly an expansion in powers of $\phi$, since $\Delta$ is itself an infinite series in $\phi$. However, since $\Delta$ starts at the order $\phi$, we need only to calculate the terms up to the order $\Delta^{2 l}$ if we are interested in the order $\phi^{2 l}$.

Note that, although the free propagator depends on a single momentum $\boldsymbol{p} \equiv\left(p_{1}, p_{2}\right)$, the full propagator carries different momenta at its two endpoints, because of the interaction with the magnetic field. Thus, eq. (13) is replaced by

$$
G_{i j, k l}=\int_{0}^{2 \pi} \frac{d p_{1} d p_{2}}{(2 \pi)^{2}} \frac{d p_{1}^{\prime} d p_{2}^{\prime}}{(2 \pi)^{2}} e^{i\left(p_{1} i-p_{1}^{\prime} k+p_{2} j-p_{2}^{\prime} l\right)} \widetilde{G}\left(\boldsymbol{p}, \boldsymbol{p}^{\prime}\right),
$$

where $\widetilde{G}\left(\boldsymbol{p}, \boldsymbol{p}^{\prime}\right)$ is the full propagator in momentum space. Here, we define the momenta in such a way that $\boldsymbol{p}$ enters at one endpoint and $\boldsymbol{p}^{\prime}$ exits at the other endpoint of the propagator. The off-diagonal momentum components of the propagator are inherited from those of the interaction term,

$$
\begin{gathered}
\mathbf{a}^{2} \widetilde{\Delta}\left(\boldsymbol{p}, \boldsymbol{p}^{\prime}\right)=(2 \pi)^{2} h_{2}\left[e^{i p_{2}} \delta\left(p_{1}^{\prime}-p_{1}-\phi\right)+e^{-i p_{2}} \delta\left(p_{1}^{\prime}-p_{1}+\phi\right)\right. \\
\left.-2 \cos \left(p_{2}\right) \delta\left(p_{1}^{\prime}-p_{1}\right)\right] \delta\left(p_{2}^{\prime}-p_{2}\right) .
\end{gathered}
$$

With the gauge choice that we have adopted, the interactions with the magnetic field always conserve the component $p_{2}$ of the momentum. Therefore, the full propagator $\widetilde{G}\left(\boldsymbol{p}, \boldsymbol{p}^{\prime}\right)$ is itself proportional to $\delta\left(p_{2}^{\prime}-p_{2}\right)$. In contrast, the magnetic field can change the component $p_{1}$, but only in discrete increments $\pm \phi$ (or 0 if we pick the third term in eq. (17)). For bookkeeping purposes, it is useful to write $\widetilde{\Delta}$ as a sum of three terms,

$$
\begin{aligned}
& \widetilde{\Delta} \equiv \sum_{\epsilon \in\{-1,0,+1\}} \widetilde{\Delta}_{\epsilon} \\
& \mathbf{a}^{2} \widetilde{\Delta}_{\epsilon} \equiv(2 \pi)^{2} h_{2} \delta\left(p_{1}^{\prime}-\left(p_{1}+\epsilon \phi\right)\right) \delta\left(p_{2}^{\prime}-p_{2}\right) V_{\epsilon}\left(p_{2}\right) \\
& V_{ \pm 1}\left(p_{2}\right) \equiv e^{ \pm i p_{2}} \quad, \quad V_{0}\left(p_{2}\right) \equiv-2 \cos \left(p_{2}\right) .
\end{aligned}
$$

The index $\epsilon$ denotes the increment (in units of $\phi$ ) of the momentum $p_{1}$ caused by the interaction. Note that

$$
V_{-1}+V_{0}+V_{+1}=0
$$

which implies that each insertion of the interaction term increases the order in $\phi$ by at least one unit. 


\subsection{Term of order $\Delta^{k}$}

We can organize the expression of $\widetilde{G}^{(k)}$ as follows,

$$
\begin{aligned}
\widetilde{G}^{(k)} & =\sum_{\left(\epsilon_{1}, \cdots, \epsilon_{k}\right) \in\{-1,0,+1\}^{k}} \widetilde{G}^{\left(k ; \epsilon_{1} \cdots \epsilon_{k}\right)} \\
\widetilde{G}^{\left(k ; \epsilon_{1} \cdots \epsilon_{k}\right)} & \equiv \widetilde{G}^{(0)} \widetilde{\Delta}_{\epsilon_{1}} \widetilde{G}^{(0)} \widetilde{\Delta}_{\epsilon_{2}} \cdots \widetilde{\Delta}_{\epsilon_{k}} \widetilde{G}^{(0)}
\end{aligned}
$$

where $\widetilde{G}^{(0)}$ is the Fourier transform of the free propagator, that can be read off from eq. (13). Tracking explicitly the momentum flow, this can be written as

$$
\widetilde{G}^{\left(k ; \epsilon_{1} \cdots \epsilon_{k}\right)}=h_{2}^{k} \widetilde{G}^{(0)}(\boldsymbol{p}) V_{\epsilon_{1}}\left(p_{2}\right) \widetilde{G}^{(0)}\left(\boldsymbol{p}+\sigma_{1} \boldsymbol{\phi}\right) \cdots V_{\epsilon_{k}}\left(p_{2}\right) \widetilde{G}^{(0)}\left(\boldsymbol{p}+\sigma_{k} \boldsymbol{\phi}\right),
$$

where we denote

$$
\phi \equiv(\phi, 0) \quad, \quad \sigma_{i} \equiv \sum_{j=1}^{i} \epsilon_{j} \quad\left(\text { and } \sigma_{0} \equiv 0\right) .
$$

The contribution of this term to the left hand side of eq. (5) reads

$$
\begin{aligned}
& G_{00,00}^{\left(k ; \epsilon_{1} \cdots \epsilon_{k}\right)}=\frac{\mathbf{a}^{2}}{4} \int \frac{d p_{1} d p_{2}}{(2 \pi)^{2}} \frac{4 h_{2}^{k}}{4-2\left(h_{1} \cos \left(p_{1}\right)+h_{2} \cos \left(p_{2}\right)\right)} \\
& \times \frac{V_{\epsilon_{1}}\left(p_{2}\right)}{4-2\left(h_{1} \cos \left(p_{1}+\sigma_{1} \phi\right)+h_{2} \cos \left(p_{2}\right)\right)} \cdots \frac{V_{\epsilon_{k}}\left(p_{2}\right)}{4-2\left(h_{1} \cos \left(p_{1}+\sigma_{k} \phi\right)+h_{2} \cos \left(p_{2}\right)\right)} .
\end{aligned}
$$

In order to make the connection with eq. (5), let us expand the integrand in powers of $h_{1,2}$ :

$$
\begin{aligned}
& \text { Integrand }=(-1)^{n_{0}} \sum_{\substack{\left(a_{0}, \cdots, a_{k}, b_{0}, \cdots, b_{k}\right) \in \mathbb{N}^{2 k+2}}} \frac{\left(a_{0}+b_{0}\right) !}{a_{0} ! b_{0} !} \cdots \frac{\left(a_{k}+b_{k}\right) !}{a_{k} ! b_{k} !} \\
& \quad \times\left(\frac{h_{1}}{4}\right)^{a_{0}+\cdots+a_{k}}\left(\frac{h_{2}}{4}\right)^{k+b_{0}+\cdots+b_{k}}\left(2 \cos \left(p_{2}\right)\right)^{b_{0}+\cdots+b_{k}+n_{0}} e^{i\left(n_{+}-n_{-}\right) p_{2}} \\
& \times\left(2 \cos \left(p_{1}\right)\right)^{a_{0}}\left(2 \cos \left(p_{1}+\sigma_{1} \phi\right)\right)^{a_{1}} \cdots\left(2 \cos \left(p_{1}+\sigma_{k} \phi\right)\right)^{a_{k}},
\end{aligned}
$$

where we denote

$$
n_{-, 0,+} \equiv \text { Card }\left\{1 \leq i \leq k \mid \epsilon_{i}=-1,0,+1\right\} .
$$

(Note that $n_{-}+n_{0}+n_{+}=k$.) The integral over $p_{2}$ is of the form

$$
\int_{0}^{2 \pi} \frac{d p_{2}}{2 \pi}\left(2 \cos p_{2}\right)^{m} e^{i n p_{2}}=\sum_{p+q=m} \frac{m !}{p ! q !} \delta_{n, p-q}=\frac{m !}{\left(\frac{m+n}{2}\right) !\left(\frac{m-n}{2}\right) !} .
$$


The final expression is valid only if $|n| \leq m$ and $m, n$ have the same parity, otherwise the integral is zero. Therefore, $m+n$ and $m-n$ should both be even. Since $m=n_{0}+\sum b_{i}$ and $n=n_{+}-n_{-}$, this implies that $k+\sum b_{i}$ should be even. This was of course expected for a closed random walk, since $k+\sum b_{i}$ is the number of steps in the directions \pm 2 (see the order in $h_{2}$ in eq. (24)). For the integral over $p_{1}$, we need

$$
\begin{aligned}
& \int_{0}^{2 \pi} \frac{d p_{1}}{2 \pi}\left(2 \cos \left(p_{1}+\sigma_{0} \phi\right)\right)^{a_{0}} \cdots\left(2 \cos \left(p_{1}+\sigma_{k} \phi\right)\right)^{a_{k}}=\sum_{\alpha_{0}+\beta_{0}=a_{0}} \cdots \\
& \cdots \sum_{\alpha_{k}+\beta_{k}=a_{k}} \frac{a_{0} !}{\alpha_{0} ! \beta_{0} !} \cdots \frac{a_{k} !}{\alpha_{k} ! \beta_{k} !} \delta_{\alpha_{0}+\cdots+\alpha_{k}, \beta_{0}+\cdots+\beta_{k}} e^{i \phi\left(\sigma_{0}\left(\alpha_{0}-\beta_{0}\right)+\cdots+\sigma_{k}\left(\alpha_{k}-\beta_{k}\right)\right)}
\end{aligned}
$$

Wrapping everything together, we obtain

$$
\begin{aligned}
& G_{00,00}^{\left(k ; \epsilon_{1} \cdots \epsilon_{k}\right)}=\frac{\mathbf{a}^{2}}{4}(-1)^{n_{0}} \sum_{n_{1}, n_{2}} \sum_{\begin{array}{c}
\alpha_{0}+\cdots+\alpha_{k}=n_{1} \\
\beta_{0}+\cdots+\beta_{k}=n_{1} \\
k+b_{0}+\cdots+b_{k}=2 n_{2}
\end{array}} \frac{\left(\alpha_{0}+\beta_{0}+b_{0}\right) !}{\alpha_{0} ! \beta_{0} ! b_{0} !} \cdots \frac{\left(\alpha_{k}+\beta_{k}+b_{k}\right) !}{\alpha_{k} ! \beta_{k} ! b_{k} !} \\
& \quad \times\left(\frac{h_{1}}{4}\right)^{2 n_{1}}\left(\frac{h_{2}}{4}\right)^{2 n_{2}} \frac{\left(2 n_{2}-n_{+}-n_{-}\right) !}{\left(n_{2}-n_{+}\right) !\left(n_{2}-n_{-}\right) !} e^{i \phi\left(\sigma_{0}\left(\alpha_{0}-\beta_{0}\right)+\cdots+\sigma_{k}\left(\alpha_{k}-\beta_{k}\right)\right)},
\end{aligned}
$$

where we have introduced

$$
2 n_{1} \equiv \sum_{i=0}^{k} a_{i} \quad, \quad 2 n_{2} \equiv k+\sum_{i=0}^{k} b_{i} .
$$

The sum over the $b_{i}$ 's can be performed easily by using the following identity (see the appendix A.1)

$$
\sum_{b_{0}+\cdots+b_{k}=B} \frac{\left(a_{0}+b_{0}\right) !}{b_{0} !} \cdots \frac{\left(a_{k}+b_{k}\right) !}{b_{k} !}=a_{0} ! \cdots a_{k} ! \frac{(A+B+k) !}{B !(A+k) !},
$$

where $A \equiv a_{0}+\cdots+a_{k}$. Therefore, eq. (28) can be rewritten as

$$
G_{00,00}^{\left(k ; \epsilon_{1} \cdots \epsilon_{k}\right)}=\frac{\mathbf{a}^{2}}{4} \sum_{n_{1}, n_{2}}\left(\frac{h_{1}}{4}\right)^{2 n_{1}}\left(\frac{h_{2}}{4}\right)^{2 n_{2}} \frac{\left(2\left(n_{1}+n_{2}\right)\right) !}{n_{1} !^{2} n_{2} !^{2}} F_{k ; \epsilon_{1} \cdots \epsilon_{k}}\left(n_{1}, n_{2} ; \phi\right)
$$

with

$$
\begin{aligned}
& F_{k ; \epsilon_{1} \cdots \epsilon_{k}}\left(n_{1}, n_{2} ; \phi\right) \equiv(-1)^{n_{0}} \frac{n_{1} !^{2}}{\left(2 n_{1}+k\right) !} \frac{n_{2} !^{2}}{\left(2 n_{2}-k\right) !} \frac{\left(2 n_{2}-n_{+}-n_{-}\right) !}{\left(n_{2}-n_{+}\right) !\left(n_{2}-n_{-}\right) !} \\
& \times \sum_{\substack{\alpha_{0}+\cdots+\alpha_{k}=n_{1} \\
\beta_{0}+\cdots+\beta_{k}=n_{1}}} \frac{\left(\alpha_{0}+\beta_{0}\right) !}{\alpha_{0} ! \beta_{0} !} \cdots \frac{\left(\alpha_{k}+\beta_{k}\right) !}{\alpha_{k} ! \beta_{k} !} e^{i \phi\left(\sigma_{0}\left(\alpha_{0}-\beta_{0}\right)+\cdots+\sigma_{k}\left(\alpha_{k}-\beta_{k}\right)\right) .}
\end{aligned}
$$


In this formula, the sum over the $\alpha_{i}$ 's and $\beta_{i}$ 's, as well as the combinatorial factors under this sum, are symmetric under the exchange $\alpha_{i} \leftrightarrow \beta_{i}$, while the argument of the exponential is antisymmetric. Therefore, $F_{k ; \epsilon_{1} \cdots \epsilon_{k}}\left(n_{1}, n_{2} ; \phi\right)$ is even in $\phi$.

\section{Moments of the distribution of areas}

\subsection{Terms of order $\phi^{0}$}

As a check, one can first evaluate eq. (32) for $\phi=0$. For this, we need the following sum (see the appendix A.2)

$$
\sum_{\substack{\alpha_{0}+\cdots+\alpha_{k}=n_{1} \\ \beta_{0}+\cdots+\beta_{k}=n_{1}}} \frac{\left(\alpha_{0}+\beta_{0}\right) !}{\alpha_{0} ! \beta_{0} !} \cdots \frac{\left(\alpha_{k}+\beta_{k}\right) !}{\alpha_{k} ! \beta_{k} !}=\frac{\left(2 n_{1}+k\right) !}{k ! n_{1} !^{2}},
$$

which leads to

$$
F_{k ; \epsilon_{1} \cdots \epsilon_{k}}\left(n_{1}, n_{2} ; 0\right)=\frac{(-1)^{n_{0}}}{k !} \frac{n_{2} !^{2}}{\left(2 n_{2}-k\right) !} \frac{\left(2 n_{2}-n_{+}-n_{-}\right) !}{\left(n_{2}-n_{+}\right) !\left(n_{2}-n_{-}\right) !} .
$$

When summing over all the possible assignments of $\epsilon_{i}$ in $\{-1,0,+1\}$, we obtain

$$
\frac{n_{2} !^{2}}{k !\left(2 n_{2}-k\right) !} \sum_{\substack{\left(\epsilon_{1}, \cdots, \epsilon_{k}\right) \\ \in\{-1,0,+1\}^{k}}}(-1)^{n_{0}} \frac{\left(2 n_{2}-n_{+}-n_{-}\right) !}{\left(n_{2}-n_{+}\right) !\left(n_{2}-n_{-}\right) !}=0 \text { if } k \geq 1,
$$

(see the appendix A.3) that vanishes as expected since the interaction terms do not contribute to the $\phi$-independent part of the result.

\subsection{Terms of order $\phi^{2}$}

In order to obtain the terms that are quadratic in the magnetic flux, we need to calculate the sum

$$
\begin{aligned}
& \sum_{\substack{\alpha_{0}+\cdots+\alpha_{k}=n_{1} \\
\beta_{0}+\cdots+\beta_{k}=n_{1}}} \frac{\left(\alpha_{0}+\beta_{0}\right) !}{\alpha_{0} ! \beta_{0} !} \cdots \frac{\left(\alpha_{k}+\beta_{k}\right) !}{\alpha_{k} ! \beta_{k} !}[\left.\sigma_{1}\left(\alpha_{1}-\beta_{1}\right)+\cdots+\sigma_{k}\left(\alpha_{k}-\beta_{k}\right)\right]^{2}= \\
&=\sum_{\substack{\alpha_{0}+\cdots+\alpha_{k}=n_{1} \\
\beta_{0}+\cdots+\beta_{k}=n_{1}}} \frac{\left(\alpha_{0}+\beta_{0}\right) !}{\alpha_{0} ! \beta_{0} !} \cdots \frac{\left(\alpha_{k}+\beta_{k}\right) !}{\alpha_{k} ! \beta_{k} !}\left[\sum_{i=1}^{k} \sigma_{i}^{2}\left(\alpha_{i}-\beta_{i}\right)^{2}\right. \\
& \\
&\left.+\sum_{i \neq j} \sigma_{i} \sigma_{j}\left(\alpha_{i}-\beta_{i}\right)\left(\alpha_{j}-\beta_{j}\right)\right] .
\end{aligned}
$$

The terms that appear in the right hand side involve sums of one of the following types (see the appendices A.4 and A.5 ,

$$
\sum_{\substack{\alpha_{0}+\cdots+\alpha_{k}=n_{1} \\ \beta_{0}+\cdots+\beta_{k}=n_{1}}} \frac{\left(\alpha_{0}+\beta_{0}\right) !}{\alpha_{0} ! \beta_{0} !} \cdots \frac{\left(\alpha_{k}+\beta_{k}\right) !}{\alpha_{k} ! \beta_{k} !}\left(\alpha_{i}-\beta_{i}\right)^{2}=\frac{2 k n_{1}}{(k+2) !} \frac{\left(2 n_{1}+k\right) !}{n_{1} !^{2}},
$$




$$
\sum_{\substack{\alpha_{0}+\cdots+\alpha_{k}=n_{1} \\ \beta_{0}+\cdots+\beta_{k}=n_{1}}} \frac{\left(\alpha_{0}+\beta_{0}\right) !}{\alpha_{0} ! \beta_{0} !} \cdots \frac{\left(\alpha_{k}+\beta_{k}\right) !}{\alpha_{k} ! \beta_{k} !}\left(\alpha_{i}-\beta_{i}\right)\left(\alpha_{j}-\beta_{j}\right)=-\frac{2 n_{1}}{(k+2) !} \frac{\left(2 n_{1}+k\right) !}{n_{1} !^{2}},
$$

$(i \neq j$ in the second equation $)$ and we obtain

$$
\begin{gathered}
\sum_{\substack{\alpha_{0}+\cdots+\alpha_{k}=n_{1} \\
\beta_{0}+\cdots+\beta_{k}=n_{1}}} \frac{\left(\alpha_{0}+\beta_{0}\right) !}{\alpha_{0} ! \beta_{0} !} \cdots \frac{\left(\alpha_{k}+\beta_{k}\right) !}{\alpha_{k} ! \beta_{k} !}\left[\sigma_{1}\left(\alpha_{1}-\beta_{1}\right)+\cdots+\sigma_{k}\left(\alpha_{k}-\beta_{k}\right)\right]^{2}= \\
=\frac{\left(2 n_{1}+k\right) !}{n_{1} !^{2}} \frac{2 n_{1}}{(k+2) !}\left[k \sum_{i=1}^{k} \sigma_{i}^{2}-\sum_{i \neq j} \sigma_{i} \sigma_{j}\right] .
\end{gathered}
$$

Since the expansion in $\Delta^{k}$ is in fact an expansion in powers of $e^{ \pm i \phi}-1$, it is a priori obvious that the terms of order $\phi^{2}$ can only appear in the orders $k=1$ and $k=2$. This corresponds to a fairly small number of terms ( 3 for $k=1$ and $3^{2}$ for $k=2$ ) that can be summed by hand to obtain

$$
\begin{aligned}
F_{k=0}\left(n_{1}, n_{2} ; \phi\right) & =1 \\
\sum_{\epsilon_{1} \in\{-1,0,+1\}} F_{k=1 ; \epsilon_{1}}\left(n_{1}, n_{2} ; \phi\right) & =-\frac{\phi^{2}}{2 !} \frac{4 n_{1} n_{2}}{3 !}+\mathcal{O}\left(\phi^{4}\right) \\
\sum_{\left(\epsilon_{1}, \epsilon_{2}\right) \in\{-1,0,+1\}^{2}} F_{k=2 ; \epsilon_{1} \epsilon_{2}}\left(n_{1}, n_{2} ; \phi\right) & =+\frac{\phi^{2}}{2 !} \frac{8 n_{1} n_{2}}{4 !}+\mathcal{O}\left(\phi^{4}\right),
\end{aligned}
$$

so that the total up to the order $\phi^{2}$ reads

$$
\sum_{k \leq 2} \sum_{\left(\epsilon_{1}, \cdots, \epsilon_{k}\right) \in\{-1,0,+1\}^{k}} F_{k ; \epsilon_{1} \cdots \epsilon_{k}}\left(n_{1}, n_{2} ; \phi\right)=1-\frac{\phi^{2}}{2 !} \frac{n_{1} n_{2}}{3}+\mathcal{O}\left(\phi^{4}\right) .
$$

From this formula, we can read out the second moment of the distribution of areas,

$$
\sum_{\gamma \in \boldsymbol{\Gamma}_{n_{1}, n_{2}}}(\text { Area }(\gamma))^{2}=\frac{\left(2\left(n_{1}+n_{2}\right)\right) !}{n_{1} !^{2} n_{2} !^{2}} \frac{n_{1} n_{2}}{3},
$$

which ends the proof of the first of eqs. (2). 


\subsection{Moment of order $2 l$}

\subsubsection{General structure}

Let us now consider the general case of the terms of arbitrary (but even) order $\phi^{2 l}$. Now, the sum we must calculate is

$$
\begin{gathered}
\sum_{\substack{\alpha_{0}+\cdots+\alpha_{k}=n_{1} \\
\beta_{0}+\cdots+\beta_{k}=n_{1}}} \frac{\left(\alpha_{0}+\beta_{0}\right) !}{\alpha_{0} ! \beta_{0} !} \cdots \frac{\left(\alpha_{k}+\beta_{k}\right) !}{\alpha_{k} ! \beta_{k} !}\left[\sigma_{1}\left(\alpha_{1}-\beta_{1}\right)+\cdots+\sigma_{k}\left(\alpha_{k}-\beta_{k}\right)\right]^{2 l}= \\
=\sum_{l_{1}+\cdots+l_{k}=2 l} \frac{(2 l) !}{l_{1} ! \cdots l_{k} !} \sigma_{1}^{l_{1}} \cdots \sigma_{k}^{l_{k}} \sum_{\substack{\alpha_{0}+\cdots+\alpha_{k}=n_{1} \\
\beta_{0}+\cdots+\beta_{k}=n_{1}}}^{\frac{\left(\alpha_{0}+\beta_{0}\right) !}{\alpha_{0} ! \beta_{0} !} \cdots \frac{\left(\alpha_{k}+\beta_{k}\right) !}{\alpha_{k} ! \beta_{k} !} \prod_{i=1}^{k}\left(\alpha_{i}-\beta_{i}\right)^{l_{i}}} . \\
\mathcal{C}_{l_{1} \cdots l_{k}}\left(n_{1}\right)
\end{gathered}
$$

In order to evaluate the sum underlined in the last line, we proceed as in the appendix A.4. First, we define a generating function

$$
H_{l_{1} \cdots l_{k}}(x, y) \equiv \sum_{n_{1}, n_{1}^{\prime} \geq 0} x^{n_{1}} y^{n_{1}^{\prime}} \sum_{\substack{\alpha_{0}+\cdots+\alpha_{k}=n_{1} \\ \beta_{0}+\cdots+\beta_{k}=n_{1}^{\prime}}} \frac{\left(\alpha_{0}+\beta_{0}\right) !}{\alpha_{0} ! \beta_{0} !} \cdots \frac{\left(\alpha_{k}+\beta_{k}\right) !}{\alpha_{k} ! \beta_{k} !} \prod_{i=1}^{k}\left(\alpha_{i}-\beta_{i}\right)^{l_{i}} .
$$

The desired quantity is the coefficient of $(x y)^{n_{1}}$ in the Taylor expansion of this function. Following the appendices A.4 and A.5 this function is also equal to

$$
H_{l_{1} \cdots l_{k}}(x, y)=\frac{1}{1-x-y} \prod_{i=1}^{k}\left[\left(x \partial_{x}-y \partial_{y}\right)^{l_{i}} \frac{1}{1-x-y}\right] \text {. }
$$

After evaluating the derivatives, the generating function takes the form

$$
H_{l_{1} \cdots l_{k}}(x, y)=\frac{A_{l_{1}}(x, y) A_{l_{2}}(x, y) \cdots A_{l_{k}}(x, y)}{(1-x-y)^{1+k+2 l}},
$$

where the $A_{n}(x, y)$ are polynomials defined iteratively by

$$
\begin{aligned}
A_{0}(x, y) & =1 \\
A_{n+1}(x, y) & =\left[(n+1)(x-y)+(1-x-y)\left(x \partial_{x}-y \partial_{y}\right)\right] A_{n}(x, y) .
\end{aligned}
$$

The degree of $A_{n}$ is thus equal to $n$, and the product in the numerator of eq. (46) can be expanded as

$$
\prod_{i=1}^{k} A_{l_{i}}(x, y)=\sum_{m+n \leq 2 l} A_{m n}\left(\left\{l_{i}\right\}\right) x^{m} y^{n} .
$$

Each term in this sum gives the following contribution to the coefficient of $(x y)^{n_{1}}$ in the Taylor expansion of the generating function :

$$
\frac{x^{m} y^{n}}{(1-x-y)^{1+k+2 l}}=\cdots+\frac{\left.\left(2 n_{1}+k+2 l-m-n\right)\right) !}{(k+2 l) !\left(n_{1}-m\right) !\left(n_{1}-n\right) !}(x y)^{n_{1}}+\cdots
$$


Let us combine $\mathcal{C}_{l_{1} \ldots l_{k}}\left(n_{1}\right)$ with the other factors that depend on $n_{1}$ in eq. (32), at the exception of the factor $\left(2\left(n_{1}+n_{2}\right)\right) ! /\left(n_{1} !^{2} n_{2} !^{2}\right)$,

$$
\begin{aligned}
& P_{l_{1} \cdots l_{k}}^{(k)}\left(n_{1}\right) \equiv \frac{n_{1} !^{2}}{\left(2 n_{1}+k\right) !} \mathcal{C}_{l_{1} \cdots l_{k}}\left(n_{1}\right) \\
& \quad=\frac{1}{(k+2 l) !} \sum_{m+n \leq 2 l} A_{m n}\left(\left\{l_{i}\right\}\right) \underbrace{\frac{n_{1} !}{\left(n_{1}-m\right) !}}_{\text {deg. } m} \underbrace{\frac{n_{1} !}{\left(n_{1}-n\right) !}}_{\text {deg. } n} \underbrace{\frac{\left.\left(2 n_{1}+k+2 l-m-n\right)\right) !}{\left(2 n_{1}+k\right) !}}_{\text {deg. } 2 l-m-n} .
\end{aligned}
$$

The three underlined factors are polynomials in $n_{1}$, of respective degrees $m$, $n$ and $2 l-m-n$, and therefore $P_{l_{1} \ldots l_{k}}^{(k)}\left(n_{1}\right)$ is also a polynomial in $n_{1}$ whose degree is bounded by $2 l$ (at this level of the discussion, it is not possible to see the cancellations that may reduce the final degree when we sum on $m, n$ ).

Let us now focus on the dependence on $n_{2}$, and combine all the $n_{2}$-dependent factors into the following quantity, except the factor $\left(2\left(n_{1}+n_{2}\right)\right) ! /\left(n_{1} !^{2} n_{2} !^{2}\right)$,

$$
Q_{l_{1} \cdots l_{k}}^{(k)}\left(n_{2}\right) \equiv \sum_{\substack{\left(\epsilon_{1}, \cdots, \epsilon_{k}\right) \\ \in\{-1,0,+1\}^{k}}}(-1)^{n_{0}}\left[\prod_{i=1}^{k} \sigma_{i}^{l_{i}}\right] \underbrace{\frac{n_{2} !}{\left(n_{2}-n_{+}\right) !}}_{\text {deg. } n_{+}} \underbrace{\frac{n_{2} !}{\left(n_{2}-n_{-}\right) !}}_{\text {deg. } n_{-}} \underbrace{\frac{\left(2 n_{2}-k+n_{0}\right) !}{\left(2 n_{2}-k\right) !}}_{\text {deg. } n_{0}},
$$

which is a sum of products of three polynomials in $n_{2}$, of respective degrees $n_{+}$, $n_{-}$and $n_{0}$ (the total degree is thus $k$ ). Therefore, $Q_{l_{1} \ldots l_{k}}^{(k)}\left(n_{2}\right)$ is a polynomial in $n_{2}$ whose degree is bounded by $k$ (again, cancellations that may decrease the degree when summing over the $\epsilon_{i}$ 's are beyond the reach of this argument - see the subsection 3.3.2).

In order to obtain the moment $2 l$ of the distribution of areas, we just need to sumb on $1 \leq k \leq 2 l$ and for each $k$ on the partitions $l_{1}+\cdots+l_{k}=2 l$. This leads to the following expression for the $2 l$-th moment :

$$
\sum_{\gamma \in \boldsymbol{\Gamma}_{n_{1}, n_{2}}}(\operatorname{Area}(\gamma))^{2 l}=\frac{\left(2\left(n_{1}+n_{2}\right)\right) !}{n_{1} !^{2} n_{2} !^{2}} \mathcal{P}_{2 l}\left(n_{1}, n_{2}\right),
$$

where $\mathcal{P}_{2 l}$ is a polynomial in $n_{1}, n_{2}$ defined as :

$$
\mathcal{P}_{2 l}\left(n_{1}, n_{2}\right) \equiv \sum_{\substack{1 \leq k \leq 2 l \\ l_{1}+\cdots+l_{k}=2 l}} \frac{(2 l) !}{l_{1} ! \cdots l_{k} !} P_{l_{1} \cdots l_{k}}^{(k)}\left(n_{1}\right) Q_{l_{1} \cdots l_{k}}^{(k)}\left(n_{2}\right) .
$$

The above counting only provides an upper bound $4 l$ for the total degree of this polynomial. However, the asymptotic behavior of these moments for large random loops is known, since their area grows like their perimeter: this implies

\footnotetext{
${ }^{5}$ Note that $\sigma_{1}^{l_{1}} \cdots \sigma_{k}^{l_{k}}$ is a homogeneous polynomial of degree $2 l$ in the $\epsilon_{i}$ 's. If $k>2 l$, some of the $\epsilon_{i}$ 's must be absent in each monomial of this polynomial. By summing over the three values $-1,0,+1$ of any of the missing $\epsilon_{i}$ 's, one gets zero (see the subsection 3.3.2).
} 
that the degree should in fact be exactly $2 l$. In addition, this polynomial should obviously be symmetric in $n_{1,2}$. This last property is non trivial in our approach, since the choice of the gauge in which we have represented the magnetic field breaks the manifest symmetry between the two directions of space.

\subsubsection{Degree of $Q_{l_{1} \cdots l_{k}}^{(k)}\left(n_{2}\right)$}

Starting from the expression (51) and expanding the factors $\sigma_{i}^{l_{i}}$, we see that $Q_{l_{1} \cdots l_{k}}^{(k)}\left(n_{2}\right)$ is a linear combination of the following polynomials :

$$
S_{\lambda_{1} \cdots \lambda_{k}}^{(k)}\left(n_{2}\right) \equiv \sum_{\substack{\left(\epsilon_{1}, \cdots, \epsilon_{k}\right) \\ \in\{-1,0,+1\}^{k}}}(-1)^{n_{0}}\left[\prod_{i=1}^{k} \epsilon_{i}^{\lambda_{i}}\right] \frac{n_{2} !}{\left(n_{2}-n_{+}\right) !} \frac{n_{2} !}{\left(n_{2}-n_{-}\right) !} \frac{\left(2 n_{2}-k+n_{0}\right) !}{\left(2 n_{2}-k\right) !}
$$

where the $\lambda_{i}$ are integers $\geq 0$, such that

$$
\sum_{i=1}^{k} \lambda_{i}=\sum_{i=1}^{k} l_{i}=2 l
$$

(This follows from the fact that the right hand side of eq. (51) is homogeneous in the $\epsilon_{i}$ 's.)

Firstly, one can prove that $S_{\lambda_{1} \cdots \lambda_{k}}^{(k)}\left(n_{2}\right)=0$ if any of the $\lambda_{i}$ 's is zero. Let us assume for instance that $\lambda_{k}=0$, and introduce the following notations :

$$
\widetilde{n}_{-, 0,+} \equiv \text { Card }\left\{1 \leq i \leq k-1 \mid \epsilon_{i}=-1,0,+1\right\},
$$

so that

$$
\begin{array}{lll}
n_{-}=\widetilde{n}_{-}+1, & n_{0,+}=\widetilde{n}_{0,+} & \text { if } \epsilon_{k}=-1 \\
n_{0}=\widetilde{n}_{0}+1, \quad n_{-,+}=\widetilde{n}_{-,+} & \text {if } \epsilon_{k}=0 \\
n_{+}=\widetilde{n}_{+}+1, \quad n_{-, 0}=\widetilde{n}_{-, 0} & \text { if } \epsilon_{k}=+1 .
\end{array}
$$

Then, the sum over the three values of $\epsilon_{k}$ contains a factor

$$
\begin{aligned}
& \sum_{\epsilon_{k} \in\{-1,0,+1\}}(-1)^{n_{0}} \frac{\left(2 n_{2}-n_{+}-n_{-}\right) !}{\left(n_{2}-n_{+}\right) !\left(n_{2}-n_{-}\right) !}= \\
& =(-1)^{\widetilde{n}_{0}} \frac{\left(2 n_{2}-k+\widetilde{n}_{0}\right) !}{\left(n_{2}-\widetilde{n}_{+}\right) !\left(n_{2}-\widetilde{n}_{-}\right) !}\left\{\left(n_{2}-\widetilde{n}_{-}\right)-\left(2 n_{2}-\widetilde{n}_{+}-\widetilde{n}_{-}\right)\right. \\
& \left.\quad+\left(n_{2}-\widetilde{n}_{+}\right)\right\}=0 .
\end{aligned}
$$

(Notice that $\widetilde{n}_{-}+\widetilde{n}_{0}+\widetilde{n}_{+}=k-1$.)

From now on, we need only to consider the situation where all the $\lambda_{i}$ 's are strictly positive. First, note that since the $\lambda_{i}$ 's are non-zero, we can exclude $\epsilon_{i}=0$ from the sum in eq. (54). Let us assume that $k_{\mathrm{e}}$ of the $\lambda_{i}$ 's are even $(\geq 2)$ and that $k_{\mathrm{o}}$ of them are odd $\left(k_{\mathrm{e}}+k_{\mathrm{o}}=k\right)$. Without loss of generality, we can 
assume that $\lambda_{1}, \cdots, \lambda_{k_{\mathrm{o}}}$ are odd and $\lambda_{k_{\mathrm{o}}+1}, \cdots, \lambda_{k}$ are even. If $\lambda_{k}$ is even, it is easy to check that

$$
S_{\lambda_{1} \cdots \lambda_{k}}^{(k)}\left(n_{2}\right)=\left(2 n_{2}-k+1\right) S_{\lambda_{1} \cdots \lambda_{k-1}}^{(k-1)}\left(n_{2}\right)
$$

by performing explicitly the sum over $\epsilon_{k}= \pm 1$. By iterating this formula, we can eliminate all the even $\lambda_{i}$ 's to obtain

$$
S_{\lambda_{1} \cdots \lambda_{k}}^{(k)}\left(n_{2}\right)=\frac{\left(2 n_{2}-k+k_{\mathrm{e}}\right) !}{\left(2 n_{2}-k\right) !} S_{\lambda_{1} \cdots \lambda_{k_{\mathrm{o}}}}^{\left(k_{\mathrm{o}}\right)}\left(n_{2}\right) .
$$

We need now to examine eq. (54) in the case where all the $\lambda_{i}$ 's are odd, in order to evaluate the last factor in the right hand side of the previous equation. When $\lambda$ is odd and $\epsilon= \pm 1$, we have $\epsilon^{\lambda}=\epsilon$. Therefore,

$$
\epsilon_{1}^{\lambda_{1}} \cdots \epsilon_{k_{\mathrm{o}}}^{\lambda_{\mathrm{o}}}=\epsilon_{1} \cdots \epsilon_{k_{\mathrm{o}}}=(-1)^{n_{\mathrm{o}-}}
$$

where we denote

$$
n_{\mathrm{o} \pm} \equiv \operatorname{Card}\left\{1 \leq i \leq k_{\mathrm{o}} \mid \epsilon_{i}= \pm 1\right\}
$$

Thus, we have

$$
S_{\lambda_{1} \cdots \lambda_{k_{\mathrm{o}}}}^{\left(k_{\mathrm{o}}\right)}\left(n_{2}\right)=\sum_{\left(\epsilon_{1}, \cdots, \epsilon_{k_{\mathrm{o}}}\right) \in\{-1,+1\}^{k_{\mathrm{o}}}}(-1)^{n_{\mathrm{o}-}} \frac{n_{2} !}{\left(n_{2}-n_{\mathrm{o}+}\right) !} \frac{n_{2} !}{\left(n_{2}-n_{\mathrm{o}-}\right) !} .
$$

Since the right hand side depends on the $\epsilon_{i}$ 's only via $n_{\mathrm{O} \pm}$, we can rewrite this as

$$
\begin{aligned}
S_{\lambda_{1} \cdots \lambda_{k_{\mathrm{o}}}}^{\left(k_{\mathrm{o}}\right)}\left(n_{2}\right) & =\sum_{n_{\mathrm{o}+}+n_{\mathrm{o}-}=k_{\mathrm{o}}} \frac{k_{\mathrm{o}} !}{n_{\mathrm{O}+} ! n_{\mathrm{o}-} !}(-1)^{n_{\mathrm{o}-}} \frac{n_{2} !}{\left(n_{2}-n_{\mathrm{o}+}\right) !} \frac{n_{2} !}{\left(n_{2}-n_{\mathrm{o}-}\right) !} \\
& = \begin{cases}0 & \text { if } k_{\mathrm{o}} \text { is odd } \\
(-1)^{p} \frac{(2 p) !}{p !} \frac{n_{2} !}{\left(n_{2}-p\right) !} & \text { if } k_{\mathrm{o}}=2 p \text { is even }\end{cases}
\end{aligned}
$$

Note that since $\sum_{i=1}^{k} \lambda_{i}=2 l$ is even, the number $k_{\mathrm{o}}$ of odd $\lambda_{i}$ 's is also even, and we are in the second case. By counting the powers of $n_{2}$ in eqs. (60) and (64), we see that

$$
\operatorname{deg}\left(S_{\lambda_{1} \cdots \lambda_{k}}^{(k)}\right)=k_{\mathrm{e}}+\frac{k_{\mathrm{O}}}{2} .
$$

Given our assumption that none of the $\lambda_{i}$ 's is zero, this leads th 6

$$
\operatorname{deg}\left(S_{\lambda_{1} \cdots \lambda_{k}}^{(k)}\right)=\frac{k_{\mathrm{O}}}{2}+k_{\mathrm{e}} \leq \frac{1}{2} \sum_{i=1}^{k} \lambda_{i}=l
$$

Since the polynomial $Q_{l_{1} \cdots l_{k}}^{(k)}\left(n_{2}\right)$ is a linear combination of the $S_{\lambda_{1} \cdots \lambda_{k}}^{(k)}\left(n_{2}\right)$, its degree is at most $l$. By symmetry, the maximum degree in the variable $n_{1}$ in the moment of order $2 l$ is also $l$.

\footnotetext{
${ }^{6}$ If $k \leq l$, the largest degree is realized when $\lambda_{1}, \cdots, \lambda_{k}$ are all even, so that $k_{\mathrm{o}}=0, k_{\mathrm{e}}=k$ and the degree is at most $k$ in this case. Therefore, the degree of $S_{\lambda_{1} \cdots \lambda_{k}}^{(k)}$ is in fact bounded by $\operatorname{Min}(k, l)$.
} 


\subsubsection{Explicit results for $2<2 l \leq 12$}

The proof of the general structure of the moment of order $2 l$ also provides a straightforward algorithm to calculate explicitly the polynomial $\mathcal{P}_{2 l}\left(n_{1}, n_{2}\right)$. Let us mention an important improvement over eq. (53), based on the fact that the polynomial $P_{l_{1} \cdots l_{k}}^{(k)}\left(n_{1}\right)$ is invariant under the permutations of $\left\{l_{1}, \cdots, l_{k}\right\}$. One can therefore arrange the partitions $\left\{l_{1}, \cdots, l_{k}\right\}$ into classes whose elements are identical up to a permutation, and compute $P_{l_{1} \cdots l_{k}}^{(k)}\left(n_{1}\right)$ only once for each class. For each class, the sum over the members of the class can be introduced inside the calculation of $Q_{l_{1} \cdots l_{k}}^{(k)}\left(n_{2}\right)$, where it becomes the innermost sum. For small $l$ up to $2 l=12$, it leads to the following expressions (we have arranged them in terms of the elementary symmetric polynomials) :

$$
\begin{aligned}
\mathcal{P}_{4}\left(n_{1}, n_{2}\right)= & \frac{n_{1} n_{2}\left(7 n_{1} n_{2}-\left(n_{1}+n_{2}\right)\right)}{15}, \\
\mathcal{P}_{6}\left(n_{1}, n_{2}\right)= & \frac{n_{1} n_{2}}{21}\left(31\left(n_{1} n_{2}\right)^{2}-15\left(n_{1} n_{2}\right)\left(n_{1}+n_{2}\right)\right. \\
& \left.+2\left(n_{1}+n_{2}\right)^{2}-\left(n_{1}+n_{2}\right)\right) \\
\mathcal{P}_{8}\left(n_{1}, n_{2}\right)= & \frac{n_{1} n_{2}}{15}\left(127\left(n_{1} n_{2}\right)^{3}-134\left(n_{1} n_{2}\right)^{2}\left(n_{1}+n_{2}\right)+53\left(n_{1} n_{2}\right)\left(n_{1}+n_{2}\right)^{2}\right. \\
& \left.-6\left(n_{1}+n_{2}\right)^{3}-22\left(n_{1} n_{2}\right)\left(n_{1}+n_{2}\right)+8\left(n_{1}+n_{2}\right)^{2}-3\left(n_{1}+n_{2}\right)\right), \\
\mathcal{P}_{10}\left(n_{1}, n_{2}\right)= & \frac{n_{1} n_{2}}{33}\left(2555\left(n_{1} n_{2}\right)^{4}-4778\left(n_{1} n_{2}\right)^{3}\left(n_{1}+n_{2}\right)\right. \\
& +3745\left(n_{1} n_{2}\right)^{2}\left(n_{1}+n_{2}\right)^{2}-1282\left(n_{1} n_{2}\right)\left(n_{1}+n_{2}\right)^{3} \\
& +120\left(n_{1}+n_{2}\right)^{4}-1444\left(n_{1} n_{2}\right)\left(n_{1}+n_{2}\right)+1438\left(n_{1} n_{2}\right)\left(n_{1}+n_{2}\right)^{2} \\
& -300\left(n_{1}+n_{2}\right)^{3}-503\left(n_{1} n_{2}\right)\left(n_{1}+n_{2}\right)+270\left(n_{1}+n_{2}\right)^{2} \\
& \left.-85\left(n_{1}+n_{2}\right)\right)
\end{aligned}
$$

and

$$
\begin{aligned}
\mathcal{P}_{12}\left(n_{1}, n_{2}\right)= & \frac{1414477\left(n_{1} n_{2}\right)^{6}}{1365}-\frac{197569\left(n_{1} n_{2}\right)^{5}\left(n_{1}+n_{2}\right)}{65} \\
& +\frac{5381569\left(n_{1} n_{2}\right)^{4}\left(n_{1}+n_{2}\right)^{2}}{1365}-\frac{2015366\left(n_{1} n_{2}\right)^{4}\left(n_{1}+n_{2}\right)}{1365} \\
& -\frac{1190473\left(n_{1} n_{2}\right)^{3}\left(n_{1}+n_{2}\right)^{3}}{455}+\frac{19486\left(n_{1} n_{2}\right)^{3}\left(n_{1}+n_{2}\right)^{2}}{7} \\
& -\frac{1321279\left(n_{1} n_{2}\right)^{3}\left(n_{1}+n_{2}\right)}{1365}+\frac{1082842\left(n_{1} n_{2}\right)^{2}\left(n_{1}+n_{2}\right)^{4}}{1365} \\
& -\frac{321112\left(n_{1} n_{2}\right)^{2}\left(n_{1}+n_{2}\right)^{3}}{195}+\frac{372679\left(n_{1} n_{2}\right)^{2}\left(n_{1}+n_{2}\right)^{2}}{273} \\
& -\frac{82664\left(n_{1} n_{2}\right)^{2}\left(n_{1}+n_{2}\right)}{195}-\frac{5528\left(n_{1} n_{2}\right)\left(n_{1}+n_{2}\right)^{5}}{91} \\
& +\frac{22112\left(n_{1} n_{2}\right)\left(n_{1}+n_{2}\right)^{4}}{91}-\frac{175514\left(n_{1} n_{2}\right)\left(n_{1}+n_{2}\right)^{3}}{455} \\
& +\frac{384196\left(n_{1} n_{2}\right)\left(n_{1}+n_{2}\right)^{2}}{1365}-\frac{21421\left(n_{1} n_{2}\right)\left(n_{1}+n_{2}\right)}{273}
\end{aligned}
$$


Two simple properties are satisfied by all these polynomials :

$$
\mathcal{P}_{2 l}\left(n_{1}, 0\right)=\mathcal{P}_{2 l}\left(0, n_{2}\right)=0 \quad, \quad \mathcal{P}_{2 l}(1,1)=\frac{1}{3} .
$$

The first one is a consequence of the fact that if $n_{1}$ or $n_{2}$ is zero, then all the closed paths one can construct have a vanishing area. The second one follows from the fact that for $n_{1}=n_{2}=1$, all the closed paths have area $-1,0$ or +1 , and therefore contribute equally to all the even moments.

The practical limitations of this algorithm are the growth of the number of terms that need to be summed, and the dramatic cancellations that occur among these terms: the intermediate calculations contain polynomials with rational coefficients whose representation involves very large integers, while the final result has a rather moderate complexity. This suggests that there may be a better way to organize the calculation, that would help avoiding these cancellations.

\section{Conclusions}

In this paper, we have used the worldline representation of scalar QED lattice propagators in two dimensions in order to obtain the general structure of the moments of the distribution of the areas of closed random walks that make fixed number of steps in the two directions. We find that these moments are the product of the number of such random walks, times a polynomial in the number of steps made in each direction. The derivation of this formula also provides an algorithm to obtain this polynomial explicitly (although this is practical only for moments of low order).

In this approach, one must choose a "gauge" to represent the transverse magnetic field whose flux measures the areas, and there are infinitely many ways of doing this. Although the final result - in particular the polynomials $\mathcal{P}_{2 l}$ - is gauge invariant, each gauge choice may lead to an alternative to eqs. (50), (51) and (53) for representing this polynomial, thereby potentially providing a more efficient way of computing it.

\section{Acknowledgements}

We would like to thank M. Bauer and P. Di Francesco for useful comments. This work is supported by the Agence Nationale de la Recherche project 11BS04-015-01. 


\section{A Combinatorial identities}

\section{A.1 Derivation of eq. (30)}

Let us consider the following function,

$$
F(x) \equiv \sum_{B \geq 0} x^{B} \sum_{b_{0}+\cdots+b_{k}=B} \frac{\left(a_{0}+b_{0}\right) !}{b_{0} !} \cdots \frac{\left(a_{k}+b_{k}\right) !}{b_{k} !} .
$$

By construction, the left hand side of eq. (30) is the $B$-th Taylor coefficient of $F(x)$. The sum on $B$ unconstrains the sums over $b_{0}, b_{1} \cdots b_{k}$, so that we can write

$$
F(x)=\prod_{i=0}^{k}\left(\sum_{b \geq 0} x^{b} \frac{\left(a_{i}+b\right) !}{b !}\right)=\prod_{i=0}^{k} \frac{a_{i} !}{(1-x)^{1+a_{i}}}=\frac{a_{0} ! \cdots a_{k} !}{(1-x)^{1+k+A}},
$$

where $A \equiv a_{0}+\cdots+a_{k}$. The Taylor coefficient of order $B$ can then be obtained as $F^{(B)}(0) / B$ !, which gives immediately the right hand side of eq. (30).

\section{A.2 Derivation of eq. (33)}

Consider the function

$$
G(x, y) \equiv \sum_{n_{1}, n_{1}^{\prime} \geq 0} x^{n_{1}} y^{n_{1}^{\prime}} \sum_{\substack{\alpha_{0}+\cdots+\alpha_{k}=n_{1} \\ \beta_{0}+\cdots+\beta_{k}=n_{1}^{\prime}}} \frac{\left(\alpha_{0}+\beta_{0}\right) !}{\alpha_{0} ! \beta_{0} !} \cdots \frac{\left(\alpha_{k}+\beta_{k}\right) !}{\alpha_{k} ! \beta_{k} !} .
$$

The left hand side of eq. (33) is the coefficient of $(x y)^{n_{1}}$ in the Taylor expansion of $G(x, y)$. This function can be rewritten as

$$
G(x, y)=\left(\sum_{\alpha, \beta \geq 0} x^{\alpha} y^{\beta} \frac{(\alpha+\beta) !}{\alpha ! \beta !}\right)^{1+k}=\frac{1}{(1-x-y)^{1+k}} .
$$

The right hand side of eq. (33) is then obtained as $\left.\left[\partial_{x}^{n_{1}} \partial_{y}^{n_{1}} G(x, y)\right) / n_{1} !^{2}\right]_{x, y=0}$.

\section{A.3 Derivation of eq. (35)}

In eq. (35), we need to perform the sum over the $3^{k}$ possible assignments for the indices $\epsilon_{1}, \cdots, \epsilon_{k}$. However, the summand depends on these indices only via the numbers $n_{-}, n_{0}, n_{+}$defined in eq. (25). Therefore, the sum over the $\epsilon_{i}$ 's can be rewritten as follows

$$
\sum_{\left(\epsilon_{1}, \cdots, \epsilon_{k}\right) \in\{-1,0,+1\}^{k}}(\cdots)=\sum_{n_{-}+n_{0}+n_{+}=k} \frac{k !}{n_{-} ! n_{0} ! n_{+} !}(\cdots) .
$$


(In the right hand side, the combinatorial factor counts the number of assignments of the $\epsilon_{i}$ 's that lead to a given triplet $\left(n_{-}, n_{0}, n_{+}\right)$.) Therefore, the sum that appears in the right hand side of eq. (35) is equal to

$$
\begin{gathered}
\quad \sum_{\left(\epsilon_{1}, \cdots, \epsilon_{k}\right) \in\{-1,0,+1\}^{k}}(-1)^{n_{0}} \frac{\left(2 n_{2}-n_{+}-n_{-}\right) !}{\left(n_{2}-n_{+}\right) !\left(n_{2}-n_{-}\right) !}= \\
=k ! \sum_{n_{0}=0}^{k} \frac{(-1)^{n_{0}}}{n_{0} !} \frac{\left(2 n_{2}+n_{0}-k\right) !}{n_{2} !^{2}} \sum_{n_{-} n_{+}=k-n_{0}} \frac{n_{2} !}{n_{+} !\left(n_{2}-n_{+}\right) !} \frac{n_{2} !}{n_{-} !\left(n_{2}-n_{-}\right) !} \\
=\frac{\left(2 n_{2}\right) !}{n_{2} !^{2}} \sum_{n_{0}=0}^{k}(-1)^{n_{0}} \frac{\left.k n_{2}\right) !}{n_{0} !\left(k-n_{0}\right) !\left(2 n_{2}+n_{0}-k\right) !}
\end{gathered}
$$

\section{A.4 Derivation of eq. (37)}

Consider the function

$$
H(x, y) \equiv \sum_{n_{1}, n_{1}^{\prime} \geq 0} x^{n_{1}} y^{n_{1}^{\prime}} \sum_{\substack{\alpha_{0}+\cdots+\alpha_{k}=n_{1} \\ \beta_{0}+\cdots+\beta_{k}=n_{1}^{\prime}}} \frac{\left(\alpha_{0}+\beta_{0}\right) !}{\alpha_{0} ! \beta_{0} !} \cdots \frac{\left(\alpha_{k}+\beta_{k}\right) !}{\alpha_{k} ! \beta_{k} !}\left(\alpha_{k}-\beta_{k}\right)^{2}
$$

The left hand side of eq. (37) is the coefficient of $(x y)^{n_{1}}$ in the Taylor expansion of $H(x, y)$. Using some results of the subsection A.2, we first obtain

$$
H(x, y)=\frac{1}{(1-x-y)^{k}} \sum_{\alpha, \beta \geq 0} \frac{(\alpha+\beta) !}{\alpha ! \beta !}(\alpha-\beta)^{2} x^{\alpha} y^{\beta} .
$$

The remaining sum in the right hand side of the previous equation is given by

$$
\begin{aligned}
\sum_{\alpha, \beta \geq 0} \frac{(\alpha+\beta) !}{\alpha ! \beta !}(\alpha-\beta)^{2} x^{\alpha} y^{\beta} & =\left(x \partial_{x}-y \partial_{y}\right)^{2} \sum_{\alpha, \beta \geq 0} \frac{(\alpha+\beta) !}{\alpha ! \beta !} x^{\alpha} y^{\beta} \\
& =\frac{x^{2}+y^{2}-6 x y+x+y}{(1-x-y)^{3}} .
\end{aligned}
$$

This leads to the following expression for $H(x, y)$,

$$
H(x, y)=\frac{1}{(1-x-y)^{1+k}}-\frac{3}{(1-x-y)^{2+k}}+\frac{2(1-4 x y)}{(1-x-y)^{3+k}} .
$$

From this formula, it is easy to extract the Taylor coefficient that gives the sum of eq. (37) :

$$
\begin{aligned}
& \frac{\left(2 n_{1}+k\right) !}{n_{1} !^{2} k !}-\frac{3\left(2 n_{1}+k+1\right) !}{n_{1} !^{2}(k+1) !}+\frac{2\left(2 n_{1}+k+2\right) !}{n_{1} !^{2}(k+2) !}-\frac{8\left(2 n_{1}+k\right) !}{\left(n_{1}-1\right) !^{2}(k+2) !}= \\
& \quad=\frac{2 k n_{1}}{(k+2) !} \frac{\left(2 n_{1}+k\right) !}{n_{1} !^{2}}
\end{aligned}
$$




\section{A.5 Derivation of eq. (38)}

This time, consider the function

$$
I(x, y) \equiv \sum_{n_{1}, n_{1}^{\prime} \geq 0} x^{n_{1}} y^{n_{1}^{\prime}} \sum_{\substack{\alpha_{0}+\cdots+\alpha_{k}=n_{1} \\ \beta_{0}+\cdots+\beta_{k}=n_{1}^{\prime}}} \frac{\left(\alpha_{0}+\beta_{0}\right) !}{\alpha_{0} ! \beta_{0} !} \cdots \frac{\left(\alpha_{k}+\beta_{k}\right) !}{\alpha_{k} ! \beta_{k} !}\left(\alpha_{i}-\beta_{i}\right)\left(\alpha_{j}-\beta_{j}\right) .
$$

Following the same reasoning as in the previous appendix, we have

$$
\begin{aligned}
I(x, y) & =\frac{1}{(1-x-y)^{k-1}}\left[\left(x \partial_{x}-y \partial_{y}\right) \frac{1}{1-x-y}\right]^{2} \\
& =\frac{(x-y)^{2}}{(1-x-y)^{3+k}} \\
& =\frac{1}{(1-x-y)^{1+k}}-\frac{2}{(1-x-y)^{2+k}}+\frac{1-4 x y}{(1-x-y)^{3+k}} .
\end{aligned}
$$

The coefficient of $(x y)^{n_{1}}$ in the Taylor expansion of this function is

$$
\begin{aligned}
& \frac{\left(2 n_{1}+k\right) !}{n_{1} !^{2} k !}-\frac{2\left(2 n_{1}+k+1\right) !}{n_{1} !^{2}(k+1) !}+\frac{\left(2 n_{1}+k+2\right) !}{n_{1} !^{2}(k+2) !}-\frac{4\left(2 n_{1}+k\right) !}{\left(n_{1}-1\right) !^{2}(k+2) !}= \\
& \quad=-\frac{2 n_{1}}{(k+2) !} \frac{\left(2 n_{1}+k\right) !}{n_{1} !^{2}}
\end{aligned}
$$

\section{B Link with the Hofstadter-Harper Hamiltonian}

The purpose of this appendix is to establish the "dictionnary" between our lattice QED approach and the Hofstadter-Harper Hamiltonian. Let us start from eq. (3), that defines the inverse propagator in the Landau gauge. In this gauge, the link variables that describe the background field on the lattice do not depend on the coordinate along the direction 2, and it is therefore convenient to perform a Fourier transform in this variable by writing

$$
\begin{aligned}
f_{i j} & \equiv \int_{0}^{2 \pi} \frac{d \nu}{2 \pi} e^{i \nu j} \tilde{f}_{i \nu} \\
D_{i j, k l} & \equiv \int_{0}^{2 \pi} \frac{d \nu d \nu^{\prime}}{(2 \pi)^{2}} e^{i\left(\nu j-\nu^{\prime} l\right)} \widetilde{D}_{i \nu, k \nu^{\prime}} .
\end{aligned}
$$

From eq. (3), it is immediate to obtain an explicit form for the Fourier transform of the inverse propagator :

$$
\mathbf{a}^{2} \widetilde{D}_{i \nu, k \nu^{\prime}}=2 \pi \delta\left(\nu-\nu^{\prime}\right)\left[4 \delta_{i, k}-(\underbrace{h_{1}\left(\delta_{i, k+1}+\delta_{i, k-1}\right)+2 h_{2} \cos (\phi i+\nu) \delta_{i, k}}_{H_{i k}^{\left(\phi, \nu, h_{1}, h_{2}\right)}})\right],
$$

where the underlined operator $H^{\left(\phi, \nu, h_{1}, h_{2}\right)}$ is the (anisotropic) Hofstadter-Harper Hamiltonian [10, 11] for a magnetic flux $\phi$ and a wavenumber $\nu$ in the direction 
2. The proportionality to $\delta\left(\nu-\nu^{\prime}\right)$ is specific to Landau gauge, and ensures that the inverse can be calculated separately in each $\nu$ sector. The Fourier transform $\widetilde{G}$ of the propagator is defined by

$$
\sum_{k \in \mathbb{Z}} \int_{0}^{2 \pi} \frac{d \nu^{\prime}}{2 \pi} \widetilde{D}_{i \nu, k \nu^{\prime}} \widetilde{G}_{k \nu^{\prime}, l \nu^{\prime \prime}}=2 \pi \delta\left(\nu-\nu^{\prime \prime}\right) \delta_{i, l}
$$

and it can be formally written as

$$
\widetilde{G}_{i \nu, k \nu^{\prime}}=\frac{\mathbf{a}^{2}}{4} 2 \pi \delta\left(\nu-\nu^{\prime}\right) \sum_{n=0}^{\infty}\left(\frac{H^{\left(\phi, \nu, h_{1}, h_{2}\right)}}{4}\right)_{i k}^{n}
$$

From this expression, the diagonal elements of the propagator in direct space are readily obtained, and by comparing with eq. (5) we get

$$
\sum_{n_{1}, n_{2}=0}^{\infty}\left(\frac{h_{1}}{4}\right)^{2 n_{1}}\left(\frac{h_{2}}{4}\right)^{2 n_{2}} \sum_{\gamma \in \boldsymbol{\Gamma}_{n_{1}, n_{2}}} e^{i \phi \operatorname{Area}(\gamma)}=\sum_{n=0}^{\infty} \int_{0}^{2 \pi} \frac{d \nu}{2 \pi}\left(\frac{H^{\left(\phi, \nu, h_{1}, h_{2}\right)}}{4}\right)_{i i}^{n} .
$$

Note that any value of the coordinate $i$ may be used in the right hand side of this equation, since the problem is invariant under discrete translations on the lattice.

Since the Hamiltonian $H^{(\phi, \nu)}$ is the sum of two terms respectively proportional to $h_{1}$ and $h_{2}$, eq. (88) leads to the following identity

$$
\sum_{\gamma \in \boldsymbol{\Gamma}_{n_{1}, n_{2}}} e^{i \phi \text { Area }(\gamma)}=\int_{0}^{2 \pi} \frac{d \nu}{2 \pi}\left(H_{1}+H_{2}^{(\phi, \nu)}\right)_{i i}^{2 n_{1}, 2 n_{2}}
$$

where

$$
\left(H_{1}\right)_{i k} \equiv \delta_{i, k+1}+\delta_{i, k-1} \quad, \quad\left(H_{2}^{(\phi, \nu)}\right)_{i k} \equiv 2 \cos (\phi i+\nu) \delta_{i, k}
$$

and where the notation $\left(H_{1}+H_{2}^{(\phi, \nu)}\right)^{2 n_{1}, 2 n_{2}}$ denotes the sum of all the terms with $2 n_{1}$ powers of $H_{1}$ and $2 n_{2}$ powers of $H_{2}^{(\phi, \nu)}$ in the $2\left(n_{1}+n_{2}\right)$-th power of $H_{1}+H_{2}^{(\phi, \nu)}$. The main difficulty in calculating the right hand side is that $H_{1}$ and $H_{2}^{(\phi, \nu)}$ do not commute (except in the trivial case $\phi=0$ ), and each term in this sum depends on how the $H_{2}^{(\phi, \nu)}$ 's are interspersed between the $H_{1}$ 's. The expansion rules described in the subsection 2.2 provide the necessary bookkeeping for performing this calculation. In particular, the quantities $\sigma_{i} \equiv$ $\sum_{j=1}^{i} \epsilon_{j}$ defined in eq. (22) record the cummulative effect of the $\phi$ 's up to the $i$-th factor $H_{1}$.

The main result of this paper, namely the eq. (1), does not apply directly to the left hand side of eq. (89) but to its expansion in powers of the flux $\phi$. Therefore, it provides only indirect information on the structure of the trace of the powers of the Hofstadter-Harper Hamiltonian: if one keeps track separately of the number of powers $n_{1}$ and $n_{2}$ of $H_{1}$ and $H_{2}^{(\phi, \nu)}$ respectively, then the Taylor 
coefficients of the expansion in $\phi$ are a combinatorial factor times a polynomial. The summation over all $n_{1}+n_{2}=n$ then leads to the structure established in ref. [6], namely $(2 n) !^{2} / n !^{4}$ times a rational fraction in $n$. Using a computer algebra system such as Maple and the expressions listed in eqs. (21), (67) and (68), one can obtain the explicit form of this rational fraction for the lowest order moments, but these expressions are not particularly illuminating.

Because it only applies to the expansion of eq. (89) in powers of $\phi$, it also seems difficult to connect our result to other known results about the spectrum of the Hofstadter-Harper Hamiltonian and in particular to the distinctive differences that arise depending on whether the flux is commensurate with $2 \pi$ or not. Indeed, the periodicity of the Hamiltonian in the coordinate $i$ when the flux is $2 \pi$ times a rational number is a "non perturbative" property which is only manifest if one does not expand the cosine in powers of $\phi$. Going beyond our result for the moments would require to perform the sum on the moment order $2 l$ in eq. (11) (which does not seem feasible given the fact that the polynomial $\mathcal{P}_{2 l}$ is not known explicitly for all $l$ ), or to perform the sum over the $\alpha_{i}$ 's and $\beta_{i}$ 's in eq. (32) without first expanding the exponential that contains the flux.

\section{References}

[1] T. Epelbaum, F. Gelis, B. Wu, JHEP 1506, 148 (2015).

[2] Z. Bern, D.A. Kosower, Phys. Rev. D 38, 1888 (1988).

[3] Z. Bern, D.A. Kosower, Phys. Rev. Lett. 66, 1669 (1991).

[4] M.J. Strassler, Nucl. Phys. B 385, 145 (1992).

[5] C. Schubert, Acta Phys. Polon. B 27, 3965 (1996).

[6] J.A. Mingo, A. Nica, J. Combinatorial Theory, A84, 55 (1998).

[7] C. Beguin, A. Valette, A. Zuk, J. of Geometry and Physics 21, 337 (1997).

[8] J. Bellissard, C. Camacho, A. Barelli, F. Claro, J. Phys. A: Math. Gen. 30, L707 (1997).

[9] S. Mashkevich, S. Ouvry, J. Stat. Phys. 137-1, 71 (2009).

[10] P.G. Harper, Proc. Phys. Soc. London A 68, 874 (1955).

[11] D.R. Hofstadter, Phys. Rev. B 14, 2239 (1976). 